\title{
Increased apoptosis and elevated Fas expression in circulating natural killer cells in gastric cancer patients
}

\author{
Hiroaki Saito · Seigo Takaya $\cdot$ Tomohiro Osaki • \\ Masahide Ikeguchi
}

Received: 22 June 2012/ Accepted: 2 November 2012/Published online: 22 November 2012

(c) The International Gastric Cancer Association and The Japanese Gastric Cancer Association 2012

\begin{abstract}
Background Immune cells undergo extensive apoptosis in patients with cancer, which may be related to immune evasion by cancerous cells. The present study was designed to investigate the relationship between natural killer (NK) cell apoptosis and Fas expression in gastric cancer patients. Methods NK cell apoptosis and Fas expression were evaluated by multicolor flow cytometry. Soluble Fas ligand (sFasL) was quantitated by enzyme-linked immunosorbent assay.

Results The frequency of apoptotic NK cells in gastric cancer patients was significantly higher than in normal controls $(p=0.0016)$. Moreover, their frequency was related to the progression of gastric cancer. Fas-positive NK cells were significantly more common in gastric cancer patients compared with normal controls $(p=0.034)$. Furthermore, Fas expression was closely related to the frequency of NK cell apoptosis $(r=0.6, p<0.0001)$. The frequency of tumor-infiltrating NK cell apoptosis was significantly higher than that of circulating NK cell apoptosis $(p=0.035)$. Furthermore, Fas-positive NK cells in gastric cancer tissues occurred significantly more often than in peripheral blood $(p=0.029)$. FasL concentration in gastric cancer patients was lower than that in normal controls, and the difference tended to be significant $(p=0.057)$. Apoptotic circulating NK cells significantly decreased after surgery compared to before surgery $(p=0.023)$. Furthermore, Fas expression on circulating
\end{abstract}

H. Saito $(\bowtie) \cdot$ S. Takaya · T. Osaki · M. Ikeguchi Division of Surgical Oncology, Department of Surgery, Tottori University School of Medicine, 36-1 Nishi-cho, Yonago 683-8504, Japan

e-mail: sai10@med.tottori-u.ac.jp
NK cells also significantly decreased after surgery compared with before surgery $(p=0.021)$.

Conclusions Upregulation of Fas expression on NK cells is related to increased apoptosis of circulating NK cells in gastric cancer patients.

Keywords Apoptosis · Fas · Fas ligand - Gastric cancer . Natural killer cells

\section{Introduction}

Natural killer (NK) cells are effector lymphocytes of the innate immune system that respond to several types of tumors and microbial infections by limiting their spread and subsequent tissue damage [1]. Because NK cells exhibit natural cytotoxicity against a broad range of human solid tumors in the absence of major histocompatibility complex molecules on target cells [2-5], they play an important role in host anticancer defense mechanisms in vitro [6] and in vivo [7, 8]. Nonetheless, NK cell immune responses are not sufficient to eradicate tumors in cases of clinical cancer because tumors develop a mechanism to escape host immune responses $[9,10]$. In fact, defects in NK cell activity have been found in various cancers [11].

Gastric cancer is one of the most common malignancies. Although prognoses of patients with gastric carcinoma have improved because of better diagnostic techniques and better intraoperative and postoperative care, death from gastric cancer still ranks second among all cancer deaths worldwide [12]. Decreased NK cell activity has been observed in gastric cancer patients. Furthermore, Takeuchi et al. demonstrated that lower NK cell activity was an independent prognostic indicator in gastric cancer patients, 
suggesting a potential role for NK cells in preventing progression of gastric cancer [13]. However, the mechanism responsible for defective NK cell function in gastric cancer has yet to be defined. One possible mechanism responsible for dysfunction of immune cells in cancer patients is extensive apoptosis, such as T cells $[14,15]$ and NK cells [16]. Tumor cells express FasL [17], receptorbinding cancer antigen expressed on SiSo cells (RCAS1) [18], and B7-H1 [19], which induce T-cell apoptosis. It has been suggested that the Fas/Fas ligand (Fas/FasL) system plays an important role in establishing tumors with a privileged immune status by inducing Fas-mediated apoptosis in tumor-specific lymphocytes. A number of studies have demonstrated that tumor cells express FasL, which induces Fas-mediated apoptosis in T cells [17, 20, 21]. Furthermore, we have previously demonstrated that Fas expression on $\mathrm{CD} 8+\mathrm{T}$ cells is closely related to the frequency of $\mathrm{CD} 8+\mathrm{T}$-cell apoptosis in gastric cancer patients [22]. On the other hand, Fas expression on NK cells remains relatively undetermined in patients with cancer. In the current study, we therefore sought to assess a potential role of Fas expression on NK cell apoptosis.

\section{Materials and methods}

Gastric cancer patients and normal donors

Thirty-eight patients treated at Tottori University Hospital (Yonago, Japan) and pathologically diagnosed with gastric cancer were enrolled in this study. None of the patients received radiotherapy, chemotherapy, or other medical interventions before surgery. International review boards at Tottori University approved the study, and informed consent for blood donations was obtained from all individuals. Patient characteristics are shown in Table 1. Healthy

Table 1 Clinicopathological parameters of gastric cancer patients enrolled in the current study

\begin{tabular}{lll}
\hline & Gastric cancer patients & Normal controls \\
\hline Gender & & \\
Male & 20 & 8 \\
Female & 18 & 9 \\
Age & $48-82(65.5)$ & $44-88(62.7)$ \\
Depth of invasion & & \\
T1 (early) & 18 & \\
T2/T3/T4 (advanced) & 20 & \\
Lymph node metastasis & & \\
Absent & 23 & \\
Present & 15 & \\
\hline
\end{tabular}

controls $(n=17)$ were age-matched $(62.7 \pm 16.1$ years for the controls vs. $65.5 \pm 9.9$ years for patients), and each experiment was performed in parallel for controls and patients.

Preparation of peripheral blood mononuclear cells (PBMCs)

Peripheral blood $(30 \mathrm{ml})$ was drawn from each donor before surgery and centrifuged using a Ficoll-Paque (Pharmacia, Uppsala, Sweden) gradient. In 10 of the 33 patients, $30 \mathrm{ml}$ peripheral blood was also drawn 1-2 months after surgery.

Isolation of tumor-infiltrating NK cells

Freshly excised tumor tissues were minced and digested with $1.5 \mathrm{mg} / \mathrm{ml}$ collagenase D (Wako Pure Chemical Industries, Osaka, Japan). The resulting cell suspensions were filtered through a mesh filter (BD, Franklin Lakes, NJ, USA). Because it is necessary to get a large piece of tissue sample to have enough tumor-infiltrating NK cells for flow cytometry analysis, tumor-infiltrating NK cells were not available for analysis from most early gastric cancer patients. Therefore, tumor-infiltrating NK cells were available in 7 patients of 38 patients in the current study.

Flow cytometry analysis

Fluorescence-activated cell sorting (FACS) analysis was performed on a FACSCalibur (BD Pharmingen, Franklin Lakes, NJ, USA), and cells were classified using the following antibodies: anti-CD3-PE-Cy5, anti-CD56-PE, and anti-CD95-FITC (BD Pharmingen).

Apoptosis in peripheral NK cells

The percentage of apoptotic cells was calculated by scoring annexin V-binding cells after back-gating of CD3-/ CD56+ cells. All gated mononuclear cell subpopulations were visualized on forward angle scatter/side angle scatter (FSC/SSC) dot plots. To include all apoptotic cells and avoid debris with a high SCC signal, the gate was set to include a wide boundary of mononuclear cells because apoptotic cells accumulate mainly in the lower FSC/SSC channels. A cutoff was set using unstained control cells.

Measurement of soluble Fas ligand (sFasL)

sFasL in human sera was measured by enzyme-linked immunosorbent assay (ELISA) using human Fas ligand/ TNFSF6 immunoassay (R\&D Systems, Minneapolis, MN, USA). 
Statistical analysis

Either paired $t$ tests or Mann-Whitney $U$ tests were used to determine statistical differences between groups. Correlations between NK cell apoptosis and Fas expression were analyzed using the Spearman rank correlation coefficient. $p<0.05$ was considered significant. GraphPad Prism software (GraphPad Software, La Jolla, CA, USA) was used for statistical analyses.

\section{Results}

Apoptosis of circulating NK cells in gastric cancer patients

We first determined the frequency of apoptotic circulating NK cells in both normal controls and gastric cancer patients. The frequency of apoptotic cells in gastric cancer patients $(21.3 \pm 11.6 \%)$ was significantly higher than that in normal controls $(11.2 \pm 5.2 \%)(p=0.0016$; Fig. 1). The frequency of apoptotic circulating NK cells was $17.4 \pm 9.8 \%$ and $24.8 \pm 12.2 \%$ in early and advanced gastric cancer patients, respectively, and the differences were significant between early and advanced gastric cancer patients $(p=0.037)$. Furthermore, there were significant differences in the frequency of apoptotic circulating NK cells between node-negative $(17.0 \pm 8.4 \%)$ and node-positive $(28.0 \pm 12.8 \%)$ gastric cancer patients $(p=0.0086)$.

Fas expression on NK cells in gastric cancer patients

To determine the mechanism responsible for increased apoptosis in circulating NK cells in gastric cancer patients, we compared Fas expression on NK cells obtained from gastric cancer patients with that from normal controls. The mean $( \pm \mathrm{SD})$ number of Fas-positive $\mathrm{NK}$ cells was $41.2 \pm 16.9 \%$ in gastric cancer patients compared with $29.5 \pm 13.0 \%$ in normal controls ( $p=0.034$; Fig. 2). Furthermore, Fas expression was closely related to the frequency of NK cell apoptosis ( $r=0.6, p<0.0001$; Fig. 3). These findings indicate that upregulation of Fas expression on circulating NK cells may be responsible for increased NK cell apoptosis in gastric cancer patients.
Fig. 1 a A representative fluorescence-activated cell sorting (FACS) result showing the frequency of apoptotic natural killer (NK) cells in normal controls and gastric cancer patients. The percentage of apoptotic cells was calculated by scoring annexin V-binding cells after back-gating of CD3-/CD56+ cells. b The frequency of apoptotic NK cells in gastric cancer patients was significantly higher than that in normal controls $(p=0.0016)$

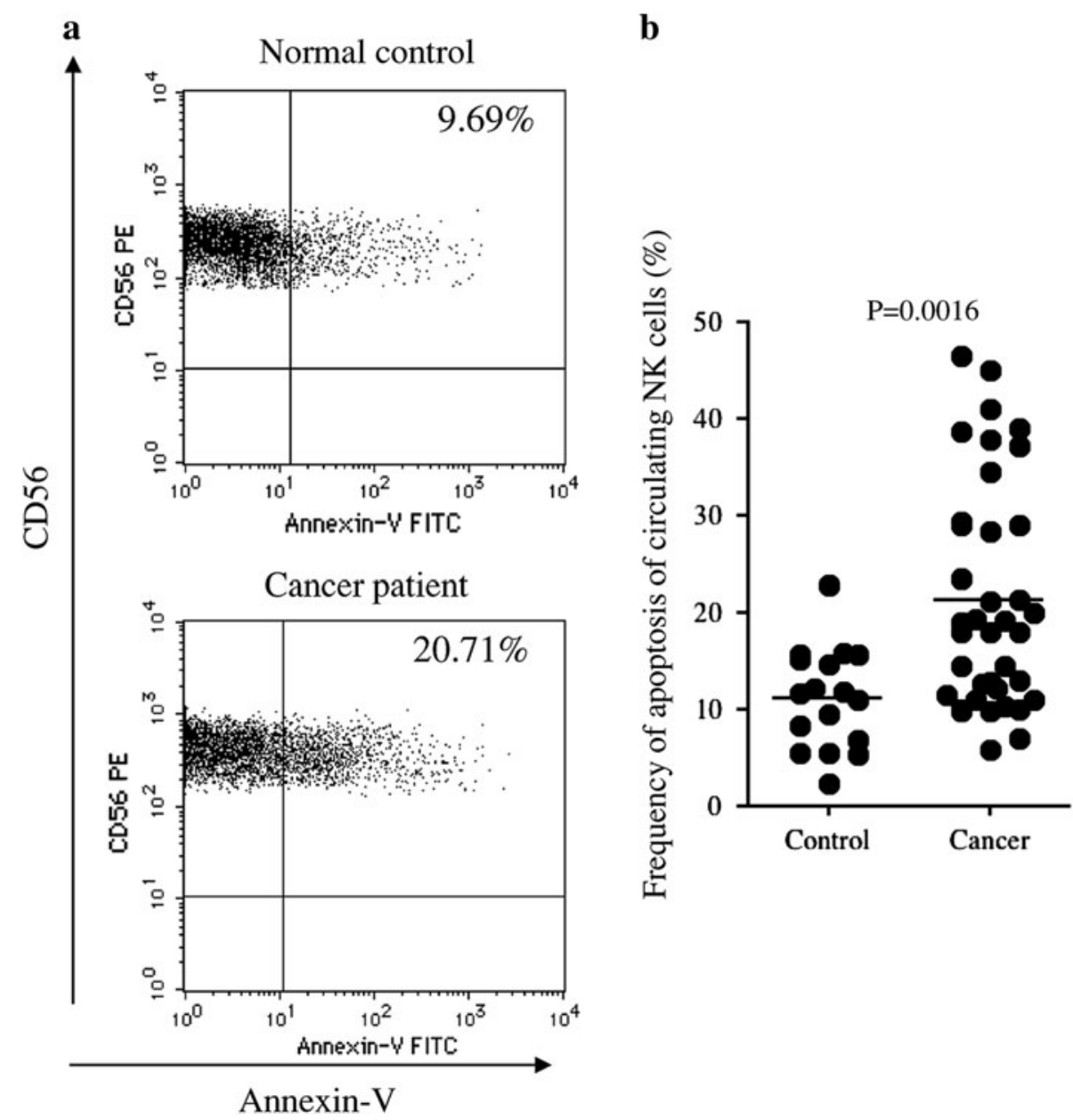


Fig. 2 a A representative FACS result showing Fas expression on NK cells from normal controls and gastric cancer patients. b Fas + NK cell frequency in gastric cancer patients was significantly higher than that in normal controls $(p=0.034)$

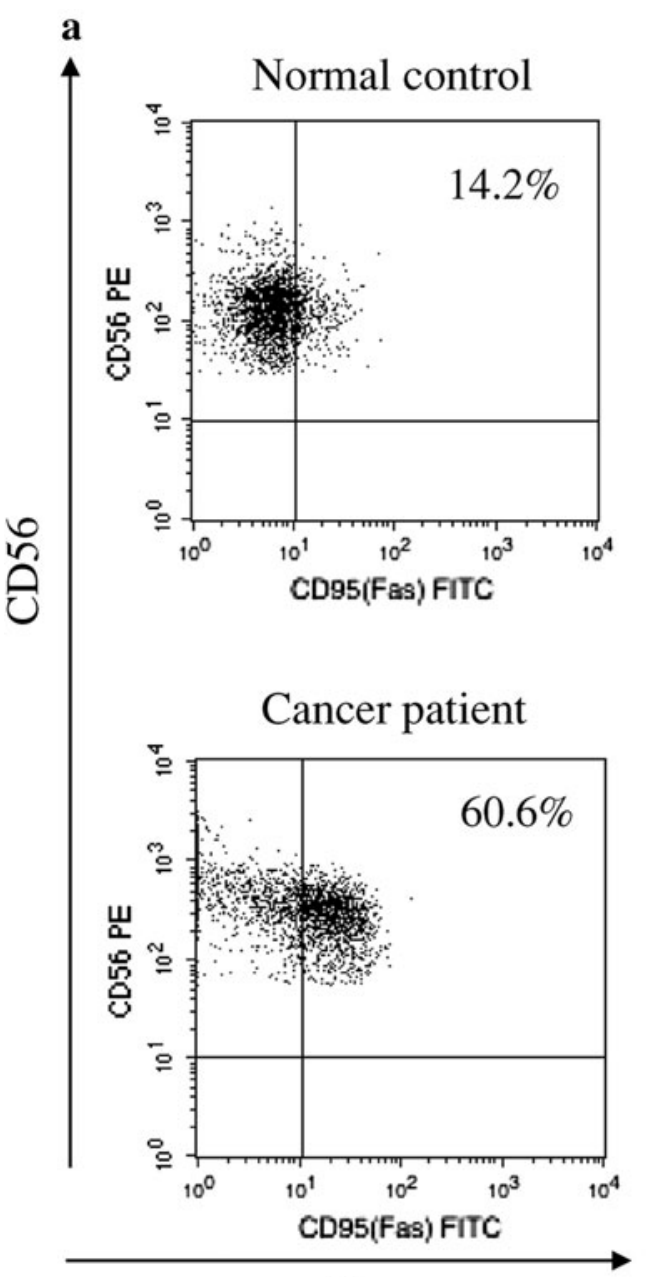

b

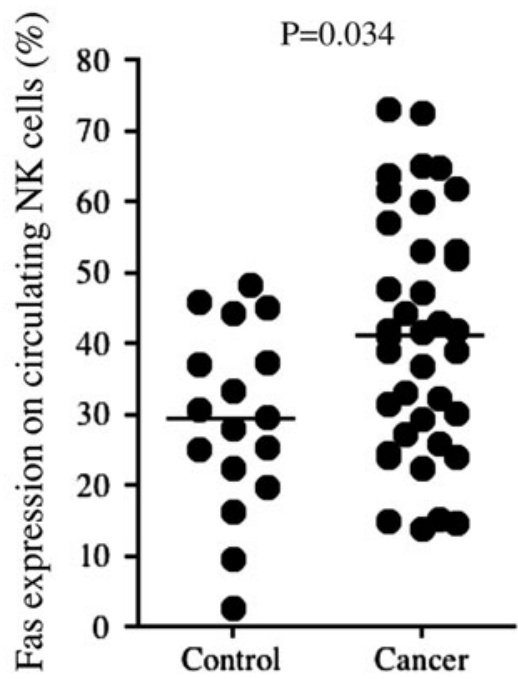

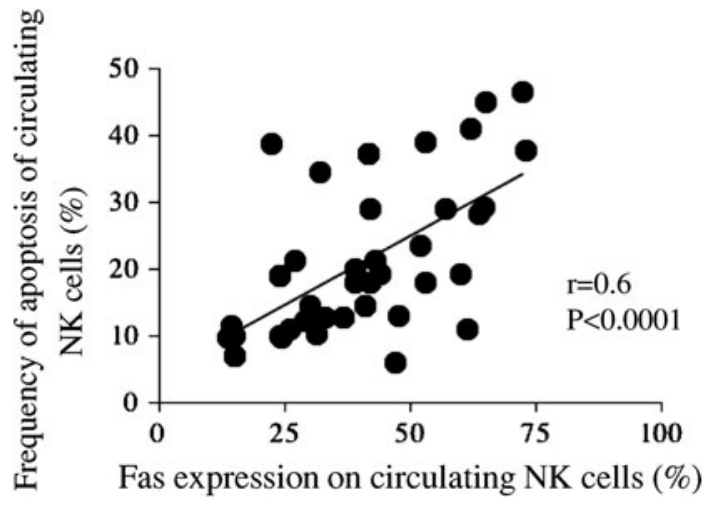

Fig. 3 The frequency of apoptotic NK cells was significantly correlated with Fas expression on NK cells $(r=0.6, p<0.0001)$

Apoptosis and Fas expression of tumor-infiltrating NK cells

We then determined the frequency of tumor-infiltrating NK cell apoptosis and found that the frequency of tumorinfiltrating $\mathrm{NK}$ cell apoptosis $(22.4 \pm 8.9 \%)$ was

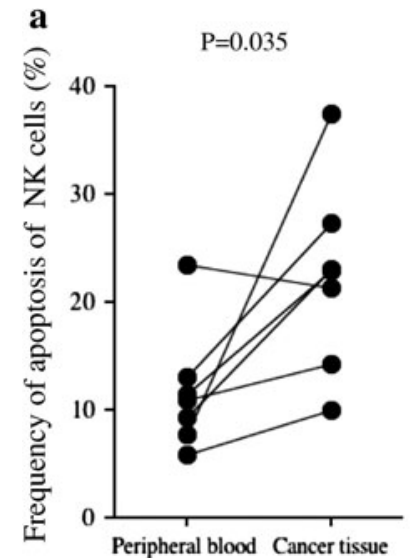

b

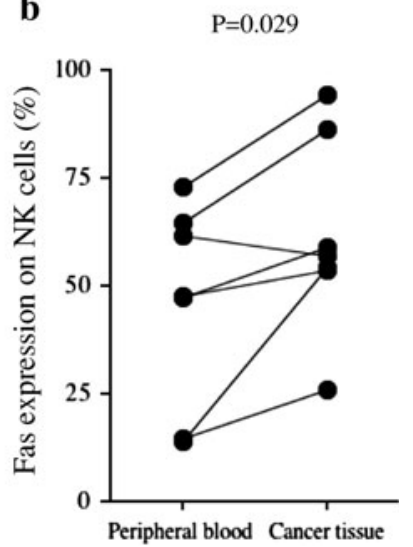

Fig. 4 a The frequency of tumor-infiltrating NK cell apoptosis was significantly higher than that of circulating NK cell apoptosis $(p=0.035)$. b Fas expression of tumor-infiltrating NK cells was significantly higher than that of circulating NK cells $(p=0.029)$

significantly higher than that of circulating NK cell apoptosis $(11.7 \pm 5.7 \%)(p=0.035$; Fig. $4 \mathrm{a})$. Furthermore, the mean $( \pm \mathrm{SD})$ number of Fas-positive NK cells was 


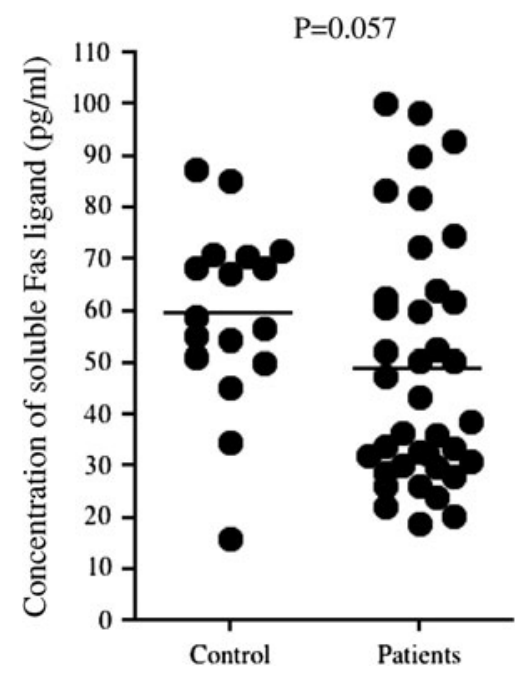

Fig. 5 The concentration of sFasL in patients with gastric cancer tended to be lower than that in normal controls $(p=0.057)$

$61.6 \pm 22.4 \%$ in tissue of gastric cancer compared with $46.2 \pm 23.6 \%$ in peripheral blood ( $p=0.029$; Fig. $4 b)$.

Serum concentrations of soluble Fas ligand in gastric cancer patients

To evaluate the contribution of the Fas/FasL pathway to circulating NK cell apoptosis, sFasL concentration was determined. Serum concentrations of sFasL were $48.9 \pm 23.7 \mathrm{pg} / \mathrm{ml}$ and $59.4 \pm 17.7 \mathrm{pg} / \mathrm{ml}$ in gastric cancer patients and controls, respectively, and the difference tended to be significant ( $p=0.057$; Fig. 5).

Decreased apoptosis and Fas expression of circulating NK cells after tumor removal

Decreased apoptosis of circulating NK cells was observed after surgery $(16.9 \pm 10.7 \%)$ compared with before surgery $(24.4 \pm 14.0 \%)(p=0.023$; Fig. 6a). Furthermore, Fas expression on circulating NK cells also significantly decreased after surgery $(24.2 \pm 9.4 \%)$ compared with before surgery $(37.4 \pm 19.7 \%)(p=0.021$; Fig. $6 b)$.

\section{Discussion}

NK cells use inhibitory receptors to monitor constitutively expressed "self" molecules on susceptible target cells. In particular, NK cells express MHC class I-specific receptors and 'lose' inhibitory signals when encountering MHC class I-deficient hematopoietic cells in several in vitro and in vivo models $[23,24]$. Because most cancer cells downregulate MHC class I expression, tumor cells are recognized as NK cell targets [1]. In fact, NK cells may
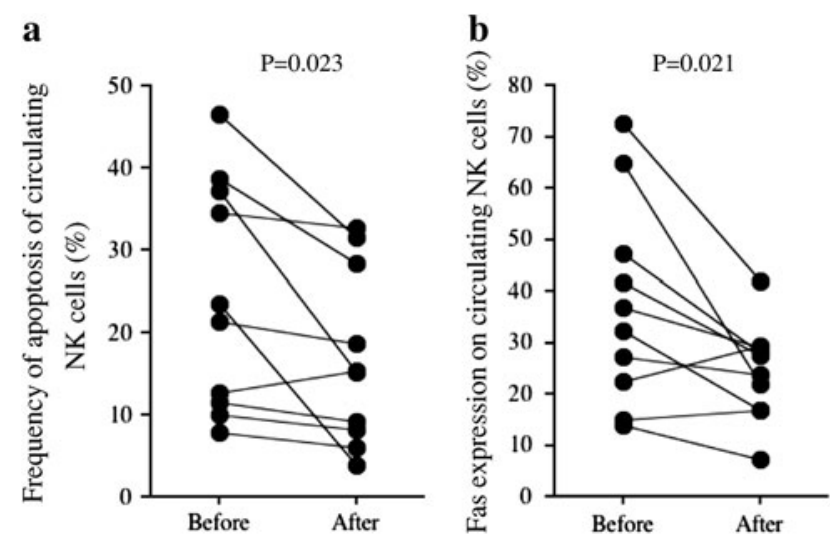

Fig. 6 a A significant decrease in the percentage of apoptotic NK cells was observed after surgery compared with that before surgery $(p=0.023)$. b A significant decrease in Fas expression on circulating NK cells was observed after surgery compared with that before surgery $(p=0.021)$. The connecting lines represent specimens from the same patient

participate in tumor immune surveillance, particularly in leukemia [25], neuroblastoma [26], and gastrointestinal stromal tumors [27]. However, NK cell function is suppressed in gastric cancer patients, even though gastric cancer cells exhibit decreased MHC class I expression [28]. This result suggests other mechanisms are responsible for the NK cell dysfunction observed in gastric cancer.

In the current study, we determined the frequency of NK cell apoptosis to assess one potential mechanism of immune evasion by gastric cancer cells. We observed more frequent NK cell apoptosis in PBMCs obtained from gastric cancer patients than from normal donors. To examine the mechanism responsible for increased apoptosis of NK cells in more detail, we examined Fas expression in these cells. Fas, a member of the tumor necrosis factor (TNF) receptor family, is a type I membrane protein expressed by a variety of cell types. Upon cross-linking by either FasL or an agonistic anti-Fas monoclonal antibody, the Fas-associated death domain and caspase- 8 form a deathinducing signaling complex; this activates the caspase cascade, resulting in apoptosis and cell death [29]. In the current study, Fas expression on circulating NK cells from gastric cancer patients was more frequent than that from normal controls. Furthermore, Fas expression was closely related to the frequency of NK cell apoptosis, indicating that increased apoptosis of circulating NK cells might be caused by elevated Fas expression.

Binding of FasL is indispensable for inducing apoptosis of Fas-positive cells. FasL is a type II transmembrane protein expressed by NK cells and activated $\mathrm{T}$ cells and within immune-privileged sites, such as the eye and brain $[30,31]$. FasL is a member of the TNF family, which also includes TNF- $\alpha$ and CD40 ligand [32]. Membrane-bound FasL is expressed on the cell surface as a $37-$ to $42-\mathrm{kDa}$ 
protein that is proteolytically cleaved by matrix metalloproteinase (MMP) to generate its $26-\mathrm{kDa}$ soluble form (sFasL) [33]. Song et al. [34] demonstrated that sFasL induces apoptosis of Fas+ T lymphocytes in patients with cancer. Moreover, studies have demonstrated that sFasL concentration in cancer patients is elevated compared with normal controls $[35,36]$. Therefore, we examined sFasL concentration in the current study. In contrast to previous reports, we observed that sFasL concentration in patients with gastric cancer trended to be lower than that in normal controls. We therefore suspected that sFasL of these patients might be consumed by binding to Fas expressed on circulating NK cells. This result is in line with a previous report on head and neck squamous cell carcinoma, demonstrating that increased lymphocytic apoptosis is associated with low-level sFasL in the serum [14]. To our knowledge, this is the first report to show that Fas upregulation on NK cells, but not an increase in sFasL concentration, correlates with apoptosis of circulating NK cells in gastric cancer patients. Fas expression significantly decreased after surgery. Furthermore, Fas expression in tumor-infiltrating NK cells was significantly higher than that in circulating NK cells. These findings indicate that gastric cancer affects Fas expression in NK cells. However, the factor responsible for upregulating Fas on NK cells remains unclear in gastric cancer patients. In this regard, we added supernatant obtained from five different gastric cancer cell lines and serum from advanced gastric cancer patients to the culture of PBMC to determine the alteration of Fas expression on NK cells and observed no alteration of Fas expression on NK cells, indicating that soluble factor including cytokines might not be responsible for the upregulation of Fas expression on NK cells (data not shown). Further investigations to clarify the mechanisms responsible for Fas upregulation on NK cells in gastric cancer patients are urgently required.

In conclusion, a significant proportion of circulating NK cells in gastric cancer patients is eliminated by apoptosis, thus potentially weakening the antitumor defense in these patients. Our results provide new data suggesting that Fas overexpression on NK cells, but not sFasL secreted from tumor cells, is related to increased apoptosis of circulating NK cells.

\section{References}

1. Trinchieri G. Biology of natural killer cells. Adv Immunol. 1989;47:187-376.

2. Tsutsui S, Morita M, Kuwano H, Matsuda H, Mori M, Okamura $\mathrm{S}$, et al. Influence of preoperative treatment and surgical operation on immune function of patients with esophageal carcinoma. J Surg Oncol. 1992;49:176-81.
3. Nunn ME, Herberman RB. Natural cytotoxicity of mouse, rat, and human lymphocytes against heterologous target cells. J Natl Cancer Inst. 1979;62:765-71.

4. Vose BM, Moore M. Natural cytotoxicity in humans: susceptibility of freshly isolated tumor cells to lysis. J Natl Cancer Inst. 1980;65:257-63.

5. Uchida A, Yanagawa E. Natural killer cell activity and autologous tumor killing activity in cancer patients: overlapping involvement of effector cells as determined in two-target conjugate cytotoxicity assay. J Natl Cancer Inst. 1984;73:1093-100.

6. Vujanovic NL, Herberman RB, Maghazachi AA, Hiserodt JC. Lymphokine-activated killer cells in rats. III. A simple method for the purification of large granular lymphocytes and their rapid expansion and conversion into lymphokine-activated killer cells. J Exp Med. 1988;167:15-29.

7. Kiessling R, Klein E, Wigzell H. "Natural" killer cells in the mouse. I. Cytotoxic cells with specificity for mouse Moloney leukemia cells. Specificity and distribution according to genotype. Eur J Immunol. 1975;5:112-7.

8. Herberman RB, Nunn ME, Lavrin DH. Natural cytotoxic reactivity of mouse lymphoid cells against syngeneic acid allogeneic tumors. I. Distribution of reactivity and specificity. Int J Cancer. $1975 ; 16: 216-29$.

9. Dunn GP, Bruce AT, Ikeda H, Old LJ, Schreiber RD. Cancer immunoediting: from immunosurveillance to tumor escape. Nat Immunol. 2002;3:991-8.

10. Whiteside TL. Immune responses to malignancies. J Allergy Clin Immunol. 2003;111:S677-86.

11. Oka M, Mitsunaga H, Hazama S, Yoshino S, Suzuki T. Natural killer activity and serum immunosuppressive acidic protein levels in esophageal and gastric cancers. Surg Today. 1993;23:669-74.

12. Ries L, Eisner M, Kosary C. SEER Cancer Statistics Review, 1975-2000. Bethesda: National Cancer Institute; 2003.

13. Takeuchi H, Maehara Y, Tokunaga E, Koga T, Kakeji Y, Sugimachi K. Prognostic significance of natural killer cell activity in patients with gastric carcinoma: a multivariate analysis. Am J Gastroenterol 2001;96:574-8.

14. Hoffmann TK, Dworacki G, Tsukihiro T, Meidenbauer N, Gooding W, Johnson JT, et al. Spontaneous apoptosis of circulating $\mathrm{T}$ lymphocytes in patients with head and neck cancer and its clinical importance. Clin Cancer Res. 2002;8:2553-62.

15. Reichert TE, Strauss L, Wagner EM, Gooding W, Whiteside TL. Signaling abnormalities, apoptosis, and reduced proliferation of circulating and tumor-infiltrating lymphocytes in patients with oral carcinoma. Clin Cancer Res. 2002;8:3137-45.

16. Bauernhofer T, Kuss I, Henderson B, Baum AS, Whiteside TL. Preferential apoptosis of CD56dim natural killer cell subset in patients with cancer. Eur J Immunol. 2003;33:119-24.

17. Gastman BR, Atarshi Y, Reichert TE, Saito T, Balkir L, Rabinowich $\mathrm{H}$, et al. Fas ligand is expressed on human squamous cell carcinomas of the head and neck, and it promotes apoptosis of $\mathrm{T}$ lymphocytes. Cancer Res. 1999;59:5356-64.

18. Nakashima M, Sonoda K, Watanabe T. Inhibition of cell growth and induction of apoptotic cell death by the human tumor-associated antigen RCAS1. Nat Med. 1999;5:938-42.

19. Dong H, Strome SE, Salomao DR, Tamura H, Hirano F, Flies DB, et al. Tumor-associated B7-H1 promotes T-cell apoptosis: a potential mechanism of immune evasion. Nat Med. 2002;8: 793-800.

20. Hahne M, Rimoldi D, Schroter M, Romero P, Schreier M, French LE, et al. Melanoma cell expression of Fas(Apo-1/CD95) ligand: implications for tumor immune escape. Science. 1996;274: 1363-6.

21. O'Connell J, O'Sullivan GC, Collins JK, Shanahan F. The Fas counterattack: Fas-mediated $\mathrm{T}$ cell killing by colon cancer cells expressing Fas ligand. J Exp Med. 1996;184:1075-82. 
22. Yoshikawa T, Saito H, Osaki T, Matsumoto S, Tsujitani S, Ikeguchi M. Elevated Fas expression is related to increased apoptosis of circulating CD8 $+\mathrm{T}$ cell in patients with gastric cancer. J Surg Res. 2008;148:143-51.

23. Yokoyama WM, Plougastel BF. Immune functions encoded by the natural killer gene complex. Nat Rev Immunol. 2003;3: 304-16.

24. Parham P. MHC class I molecules and KIRs in human history, health and survival. Nat Rev Immunol. 2005;5:201-14.

25. Ruggeri L, Aversa F, Martelli MF, Velardi A. Allogeneic hematopoietic transplantation and natural killer cell recognition of missing self. Immunol Rev. 2006;214:202-18.

26. Castriconi R, Dondero A, Corrias MV, Lanino E, Pende D, Moretta L, et al. Natural killer cell-mediated killing of freshly isolated neuroblastoma cells: critical role of DNAX accessory molecule-1-poliovirus receptor interaction. Cancer Res. 2004;64: 9180-4.

27. Borg C, Terme M, Taieb J, Menard C, Flament C, Robert C, et al. Novel mode of action of c-kit tyrosine kinase inhibitors leading to NK cell-dependent antitumor effects. J Clin Invest. 2004;114: 379-88.

28. Shen YQ, Zhang JQ, Miao FQ, Zhang JM, Jiang Q, Chen H, et al. Relationship between the downregulation of HLA class I antigen and clinicopathological significance in gastric cancer. World $\mathbf{J}$ Gastroenterol. 2005;11:3628-31.

29. Krammer PH. CD95's deadly mission in the immune system. Natur (Lond). 2000;407:789-95.
30. Stuart PM, Griffith TS, Usui N, Pepose J, Yu X, Ferguson TA. CD95 ligand (FasL)-induced apoptosis is necessary for corneal allograft survival. J Clin Invest. 1997;99:396-402.

31. Bechmann I, Mor G, Nilsen J, Eliza M, Nitsch R, Naftolin F. FasL (CD95L, Apo1L) is expressed in the normal rat and human brain: evidence for the existence of an immunological brain barrier. Glia. 1999;27:62-74.

32. Orlinick JR, Elkon KB, Chao MV. Separate domains of the human Fas ligand dictate self-association and receptor binding. J Biol Chem. 1997;272:32221-9.

33. Kayagaki N, Kawasaki A, Ebata T, Ohmoto H, Ikeda S, Inoue S, et al. Metalloproteinase-mediated release of human Fas ligand. J Exp Med. 1995;182:1777-83.

34. Song E, Chen J, Ouyang N, Su F, Wang M, Heemann U. Soluble Fas ligand released by colon adenocarcinoma cells induces host lymphocyte apoptosis: an active mode of immune evasion in colon cancer. Br J Cancer. 2001;85:1047-54.

35. Mizutani Y, Hongo F, Sato N, Ogawa O, Yoshida O, Miki T. Significance of serum soluble Fas ligand in patients with bladder carcinoma. Cancer (Phila). 2001;92:287-93.

36. Nagao M, Nakajima Y, Hisanaga M, Kayagaki N, Kanehiro H, Aomatsu Y, et al. The alteration of Fas receptor and ligand system in hepatocellular carcinomas: how do hepatoma cells escape from the host immune surveillance in vivo? Hepatology. 1999;30:413-21. 